\title{
Estimation of Sine Inverse Exponential Model under Censored Schemes
}

\author{
M. Shrahili, ${ }^{1}$ I. Elbatal, ${ }^{2}$ Waleed Almutiry $\mathbb{D}^{\mathrm{D}},{ }^{3}$ and Mohammed Elgarhy $\mathbb{D}^{4}$ \\ ${ }^{1}$ Department of Statistics and Operations Research, College of Science, King Saud University, P.O. Box 2455, \\ Riyadh 11451, Saudi Arabia \\ ${ }^{2}$ Department of Mathematics and Statistics, College of Science Imam Mohammad Ibn Saud Islamic University (IMSIU), \\ Riyadh, Saudi Arabia \\ ${ }^{3}$ Department of Mathematics, College of Science and Arts in Ar Rass, Qassim University, Ar Rass, Saudi Arabia \\ ${ }^{4}$ The Higher Institute of Commercial Sciences, Al Mahalla Al Kubra 31951, Algharbia, Egypt
}

Correspondence should be addressed to Mohammed Elgarhy; m_elgarhy85@sva.edu.eg

Received 21 August 2021; Revised 30 September 2021; Accepted 7 October 2021; Published 21 October 2021

Academic Editor: Naeem Jan

Copyright (c) $2021 \mathrm{M}$. Shrahili et al. This is an open access article distributed under the Creative Commons Attribution License, which permits unrestricted use, distribution, and reproduction in any medium, provided the original work is properly cited.

In this article, we introduce a new one-parameter model, which is named sine inverted exponential (SIE) distribution. The SIE distribution is a new extension of the inverse exponential (IE) distribution. The SIE distribution aims to provide the SIE model for data-fitting purposes. The SIE distribution is more flexible than the inverted exponential (IE) model, and it has many applications in physics, medicine, engineering, nanophysics, and nanoscience. The density function (PDFu) of SIE distribution can be unimodel shape and right skewed shape. The hazard rate function (HRFu) of SIE distribution can be J-shaped and increasing shaped. We investigated some fundamental statistical properties such as quantile function $(\mathrm{QFu})$, moments (Mo), moment generating function $(\mathrm{MGFu})$, incomplete moments (ICMo), conditional moments (CMo), and the SIE distribution parameters were estimated using the maximum likelihood (ML) method for estimation under censored samples (CS). Finally, the numerical results were investigated to evaluate the flexibility of the new model. The SIE distribution and the IE distribution are compared using two real datasets. The numerical results show the superiority of the SIE distribution.

\section{Introduction}

In the recent years, inverse and half-inverse problems are studied in general operator theory [1-3], numerous authors have attracted the attention of generated families of distributions such as Kumaraswamy-G by [4], sine generated (S-G) by [5], Kumaraswamy Type-I half-logistic-G [6], Weibull-G [7], odd Fréchet -G by [8], the Burr type X-G by [9], Kumaraswamy Kumaraswamy-G [10], truncated Cauchy power- $G$ by [11], generalized odd half-Cauchy-G by [12], and among others.

The cumulative distribution function $(\mathrm{CDFu})$ and $\mathrm{PDFu}$ of S-G are

$$
F(x ; \xi)=\sin \left[\frac{\pi}{2} G(x ; \xi)\right], \quad x \in R,
$$

$$
f(x ; \xi)=\frac{\pi}{2} g(x ; \xi) \cos \left[\frac{\pi}{2} G(x ; \xi)\right], \quad x \in R .
$$

Letting $g(x ; \xi)$ and $G(x ; \xi)$, the PDFu and CDFu of IE distribution, it has the following form:

$$
\begin{aligned}
& g(x)=\theta x^{-2} e^{-(\theta / x)}, \quad \theta>0, x>0, \\
& G(x)=e^{-(\theta / x)}, \quad \theta>0, x>0 .
\end{aligned}
$$

The main idea for this paper was to introduce a new oneparameter model that is more flexible than the IE model by using the S-G family. The new model is called the SIE model. The SIE model is more flexible than the IE model and it has 
many applications in physics, medicine, nanophysics, and nanoscience [13-16]. This manuscript is arranged as follows. Section 2 presents materials and methods. In Section 3, statistical inference of the SIE model under the censored scheme is studied. Section 4 presents results and discussion. At the end of article, conclusions are discussed.

\section{Materials and Methods}

2.1. The New SIE Model. Letting random variable $X$ to have SIE distribution, then the CDFu, PDFu, survival function (SFu), and HRFu of $X$ are

$$
\begin{aligned}
& F(x)=\sin \left[\frac{\pi}{2} e^{-(\theta / x)}\right], \quad x>0, \theta>0, \\
& f(x)=\frac{\pi \theta}{2 x^{2}} e^{-(\theta / x)} \cos \left[\frac{\pi}{2} e^{-(\theta / x)}\right], \quad x>0, \theta>0, \\
& R(x)=1-\sin \left[\frac{\pi}{2} e^{-(\theta / x)}\right], \\
& h(x)=\frac{\left(\pi \theta / 2 x^{2}\right) e^{-(\theta / x)} \cos \left[(\pi / 2) e^{-(\theta / x)}\right]}{1-\sin \left[(\pi / 2) e^{-(\theta / x)}\right]}
\end{aligned}
$$

where $\theta$ is a scale parameter.

Figures 1-3 show the plots of PDFu, CDFu, and HRFu of the SIE model. The PDFu of the SIE model can be right skewed and unimodal shaped, while the HRFu of the SIE model can be increasing or J-shaped.

2.2. Quantile and Median. If $\mathrm{X} \sim \mathrm{SIE}$, then the $\mathrm{QFu}$ of SIE is as follows:

$$
Q(u)=\theta\left[\ln \left(\frac{\pi}{2 \operatorname{Arcsin}(u)}\right)\right]^{-1},
$$

and by taking $u=0.5$, we get the median $(M)$ as $M=\theta[\ln (3)]^{-1}$.

MacGillivray's skewness function is defined in [17] as

$$
\text { MGS }=\frac{Q(u)+Q(1-u)-2 M}{Q(u)-Q(1-u)} .
$$

Figure 4 plots MGS for all values of the parameter $\theta$.

\subsection{Moments}

Theorem 1. Letting $X$ be a r.v. from the SIE model, then its $r^{\text {th }}$ Mo is

$$
\theta_{r}^{\prime}=\sum_{i=0}^{\infty} \frac{\theta^{r-1} \Lambda \Gamma(1-r)}{(2 i+1)^{1-r}}
$$

Proof. Let $X$ be a $r$. v. with pdf equation (6). The $r^{\text {th }}$ Mo of SIE distribution is computed as follows:

$$
\theta_{r}^{\prime}=\int_{0}^{\infty} x^{r} f(x) \mathrm{d} x=\frac{\pi \theta}{2} \int_{0}^{\infty} x^{r-2} e^{-(\theta / x)} \cos \left[\frac{\pi}{2} e^{-(\theta / x)}\right] \mathrm{d} x .
$$

By inserting the expansion $\cos [G(x)]=\sum_{i=0}^{\infty}\left((-1)^{i} /\right.$ (2i)!) $G(x)^{2 i}, n$ to the previous equation, then

$$
\theta_{r}^{\prime}=\sum_{i=0}^{\infty} \frac{\theta(-1)^{i}}{(2 i) !}\left(\frac{\pi}{2}\right)^{2 i+1} \int_{0}^{\infty} x^{r-\delta-1} e^{-(2 i+1)(\theta / x)} \mathrm{d} x .
$$

The last equation can be rewritten as follows:

$$
\theta_{r}^{\prime}=\sum_{i=0}^{\infty} \Lambda \int_{0}^{\infty} x^{r-2} e^{-(2 i+1)(\theta / x)} \mathrm{d} x,
$$

where $\Lambda=\sum_{i=0}^{\infty}\left(\theta(-1)^{i} /(2 i) !\right)(\pi / 2)^{2 i+1}$.

Let $y=(\theta / x)$; then,

$$
\theta_{r}^{\prime}=\sum_{i=0}^{\infty} \theta^{r-1} \Lambda \int_{0}^{\infty} y^{-r} e^{-(2 i+1) y} \mathrm{~d} y .
$$

Then,

$$
\theta_{r}^{\prime}=\sum_{i=0}^{\infty} \frac{\theta^{r-1} \Lambda \Gamma(1-r)}{(2 i+1)^{1-r}}
$$

The MGFu of $X$ is

$$
M_{X}(t)=\sum_{r=0}^{\infty} \frac{t^{r}}{r !} \theta_{r}^{\prime}=\sum_{r, i=0}^{\infty} \frac{t^{r} \theta^{r-1} \Lambda \Gamma(1-r)}{r !(2 i+1)^{1-r}} .
$$

The ICMo, denoted by $\varphi_{s}(t)$, of the SIE distribution is $\varphi_{s}(t)=\int_{0}^{t} x^{s} f(x) \mathrm{d} x=\frac{\pi \theta}{2} \int_{0}^{t} x^{s-2} e^{-(\theta / x)} \cos \left[\frac{\pi}{2} e^{-(\theta / x)}\right] \mathrm{d} x$.

By using equation (18), $\varphi_{s}(t)$ will be as

$$
\varphi_{s}(t)=\sum_{i=0}^{\infty} \frac{\theta^{r-1} \Lambda \gamma(1-r,(\theta / t))}{(2 i+1)^{1-r}},
$$

where $\gamma(s, t)=\int_{0}^{t} y^{s-1} e^{-y} \mathrm{~d} y$ is the lower ICGFu where ICGFu is incomplete gamma function.

The CMo, denoted by $\tau_{s}(t)$, of the SIE distribution is $\tau_{s}(t)=\int_{t}^{\infty} x^{s} f(x) \mathrm{d} x=\frac{\pi \theta}{2} \int_{t}^{\infty} x^{s-2} e^{-(\theta / x)} \cos \left[\frac{\pi}{2} e^{-(\theta / x)}\right] \mathrm{d} x$.

By using equation $(20), \tau_{s}(t)$ will be as given

$$
\tau_{s}(t)=\sum_{i=0}^{\infty} \frac{\theta^{r-1} \Lambda \Gamma(1-r,(\theta / t))}{(2 i+1)^{1-r}},
$$

where $\Gamma(s, t)=\int_{t}^{\infty} y^{s-1} e^{-y} \mathrm{~d} y$ is the upper ICGFu.

2.4. Order Statistics. Let $X_{1}, X_{2}, \ldots, X_{n}$ be random sample from the SIE distribution with order statistics (OS) $X_{(1)}, X_{(2)}, \ldots, X_{(n)}$. The pdf of $T_{(m)}$ of OS is 


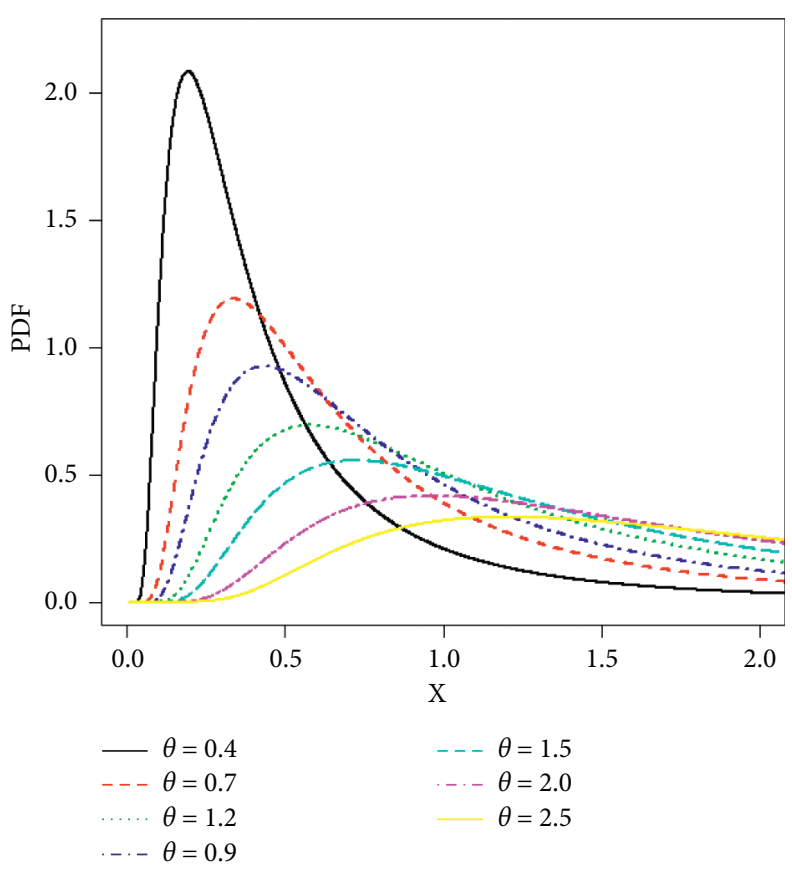

FIgURe 1: Plots of PDFu for the SIE model.

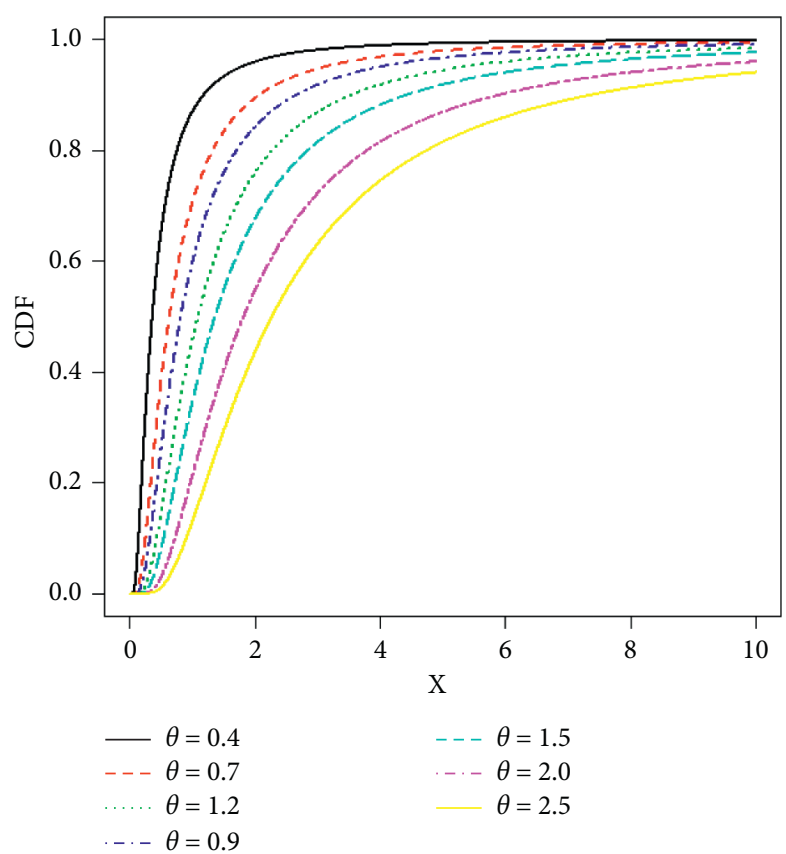

FIgURE 2: Plots of CDFu for the SIE model.

$f_{X_{(m)}}(x)=\frac{n !}{(m-1) !(n-m) !} F^{m-1}(x) f(x)(1-F(x))^{n-m}$.

The pdf of $X_{(m)}$ can be expressed as

$$
f_{X_{(m)}}(x)=\frac{n ! \pi \theta}{2(m-1) !(n-m) !} x^{-2} e^{-(\theta / x)} \sin ^{m-1}\left[\frac{\pi}{2} e^{-(\theta / x)}\right] \cos \left[\frac{\pi}{2} e^{-(\theta / x)}\right]\left(1-\sin \left[\frac{\pi}{2} e^{-(\theta / x)}\right]\right)^{n-m} .
$$




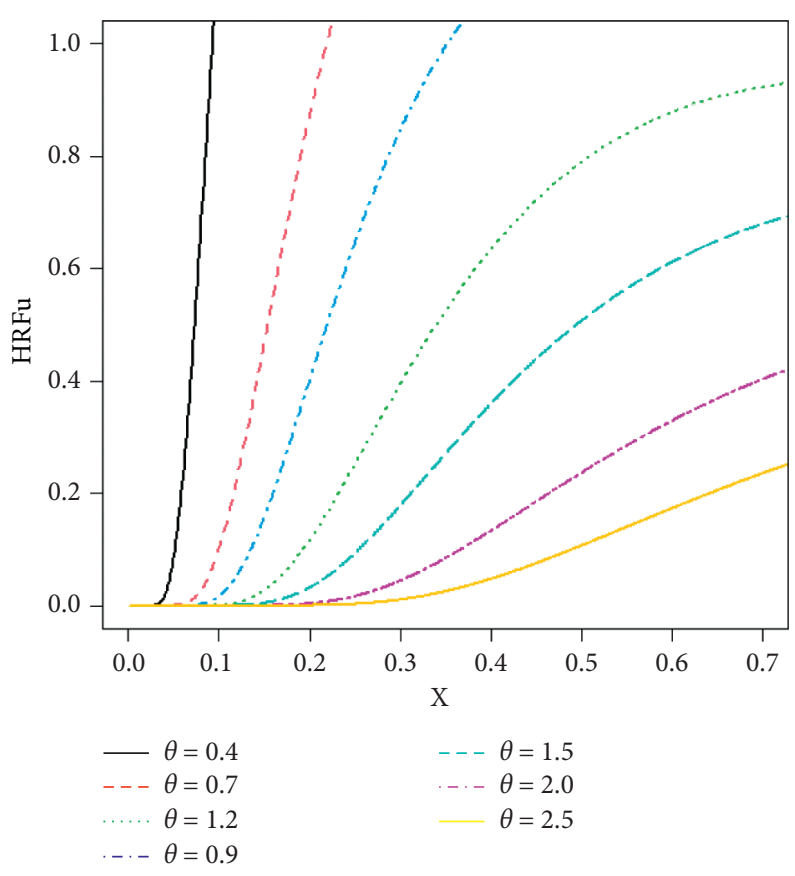

FIgUre 3: Plots of HRFu for the SIE model.

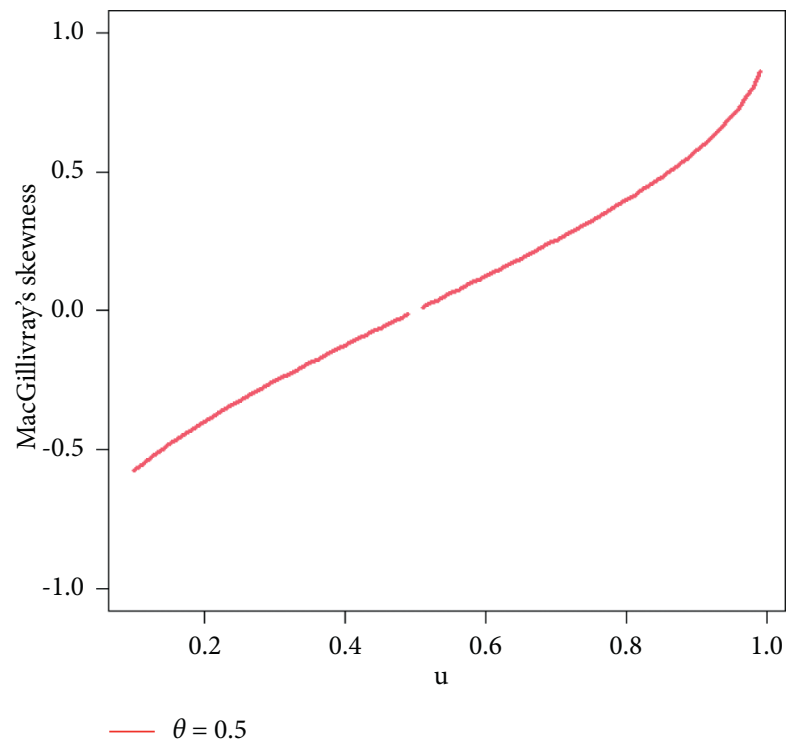

FIGURE 4: Plots of MGS for the SIE model.

Specially, the pdfs of the lowest and greatest OS can be computed as

$$
f_{X_{(1)}}(x)=\frac{n \pi \theta}{2} x^{-2} e^{-(\theta / x)} \cos \left[\frac{\pi}{2} e^{-(\theta / x)}\right]\left(1-\sin \left[\frac{\pi}{2} e^{-(\theta / x)}\right]\right)^{n-1},
$$

$f_{X_{(n)}}(x)=\frac{n \pi \theta}{2} x^{-2} e^{-(\theta / x)} \sin ^{n-1}\left[\frac{\pi}{2} e^{-(\theta / x)}\right] \cos \left[\frac{\pi}{2} e^{-(\theta / x)}\right]$.

\section{Statistical Inference under Censored Samples}

For different reasons, such as time constraints, money, or other resources, reliability or lifespan testing trials are typically censored. Generally speaking, there are two types of censorship schemes: Type-I and Type-II CS. Estimation using these two censoring techniques will be discussed in this section of the paper. If we use type-I censoring, we have a set time, say $X$, but the amount of things that fail during the trial is completely random. Type-II censoring, on the contrary, is a process that continues until the stated number of failures is reached. 
3.1. ML Estimation under Type-I Censor. Assume that $X_{1}, X_{2}, \ldots, X_{r}$ be a type-I CS of size $r$ obtained from lifetime testing experiment on $k$ items whose lifetime follows the PDFu for SIE. The likelihood function (LLFu) of type-I CS is given as

$$
L=\frac{n !}{(n-r) !}\left[1-F\left(x_{0}\right)\right]^{n-r}\left\{\prod_{i=1}^{r}\left[f\left(x_{i}\right)\right]\right\} .
$$

The log-LLFu corresponding to equation (26) is given by

$$
\ln (L)=\ln \left(\frac{n !}{(n-r) !}\right)+(n-r) \ln \left(1-\sin \left[\frac{\pi}{2} e^{-\left(\theta / x_{0}\right)}\right]\right)+r \ln \frac{\pi}{2}+r \ln \theta-2 \sum_{i=1}^{r} \ln \left(x_{i}\right)-\sum_{i=1}^{r} \frac{\theta}{x_{i}}+\sum_{i=1}^{r} \ln \left(\cos \left[\frac{\pi}{2} e^{-\left(\theta / x_{i}\right)}\right]\right) .
$$

The ML equations for the SIE distribution are as follows:

$$
\frac{\partial \ln L}{\partial \theta}=\frac{(n-r) \pi e^{-\left(\theta / x_{0}\right)} \cos \left[(\pi / 2) e^{-\left(\theta / x_{0}\right)}\right]}{2 x_{0}\left[1-\sin \left[(\pi / 2) e^{-\left(\theta / x_{0}\right)}\right]\right]}+\frac{r}{\theta}-\sum_{i=1}^{r} \frac{\theta}{x_{i}}+\frac{\delta \pi}{2 \theta} \sum_{i=1}^{r}\left(\frac{\theta}{x_{i}}\right)^{\delta} e^{-\left(\theta / x_{i}\right)^{\delta}}\left(\tan \left[\frac{\pi}{2} e^{-\left(\theta / x_{i}\right)^{\delta}}\right]\right) .
$$

Then, the ML estimators for the parameter $\theta$ are computed by putting $(\partial \ln L / \partial \theta)=0$ and solving.

3.2. ML Estimation under Type-II Censor. Let $X_{1}, X_{2}, \ldots, X_{r}$ be a type-II CS of size $r$ observed from lifetime testing experiment on $k$ items whose lifetime has the PDFu for SIE.
The LLFu of type-II CS is

$$
L=\frac{n !}{(n-r) !}\left[1-F\left(x_{r}\right)\right]^{n-r}\left\{\prod_{i=1}^{r}\left[f\left(x_{i}\right)\right]\right\} .
$$

The log-LLFu corresponding to equation (29) is given by

$\ln (L)=\ln \left(\frac{n !}{(n-r) !}\right)+(n-r) \ln \left(1-\sin \left[\frac{\pi}{2} e^{-\left(\theta / x_{r}\right)}\right]\right)+r \ln \frac{\pi}{2}+r \ln \theta-2 \sum_{i=1}^{r} \ln \left(x_{i}\right)-\sum_{i=1}^{r} \frac{\theta}{x_{i}}+\sum_{i=1}^{r} \ln \left(\cos \left[\frac{\pi}{2} e^{-\left(\theta / x_{i}\right)}\right]\right)$.

The ML equations for the SIE distribution are as follows:

$$
\frac{\partial \ln L}{\partial \theta}=\frac{(n-r) \pi e^{-\left(\theta / x_{r}\right)} \cos \left[(\pi / 2) e^{-\left(\theta / x_{r}\right)}\right]}{2 x_{r}\left[1-\sin \left[(\pi / 2) e^{-\left(\theta / x_{r}\right)}\right]\right]}+\frac{r}{\theta}-\sum_{i=1}^{r} \frac{\theta}{x_{i}}+\frac{\delta \pi}{2 \theta} \sum_{i=1}^{r}\left(\frac{\theta}{x_{i}}\right)^{\delta} e^{-\left(\theta / x_{i}\right)^{\delta}}\left(\tan \left[\frac{\pi}{2} e^{-\left(\theta / x_{i}\right)^{\delta}}\right]\right) .
$$

Then, the ML estimators for the parameter $\theta$ is calculated by putting $(\partial \ln L / \partial \theta)=0$ and solving.

3.3. Simulation Outcomes. Numerical outcomes are given in this section to examine how the estimators behave in cases of full, TIC, and TIIC estimations. With the help of Mathematica 9 , you can compute the mean square errors $(A 1)$ as well as the lower limit $(L 1)$ and upper bound (U1) of the confidence interval, as well as the average length (AvLe) for 90 percent and 95 percent. The following is a description of how the following algorithm works:

(i) SIE distribution generates 5000 random samples with size $n=30,50,100,200,300,500,1000$, and 2000. (ii) True parameter $\theta$ values are taken.

(iii) The termination time is set to $T=1.5$ in the event of TIC and 3 in the absence of TIC. Three levels of censorship are chosen: $r=80 \%, r=90 \%$ (TIIC), and $r=100 \%$ (complete sample).

(iv) The ML estimates, $A 1, L 1, U 1$, and AvLe for various parameter values are computed.

(v) Tables 1-6 include numerical outputs based on completeness and TIIC, while Tables 7-10 contain TIC-based simulation findings.

From Tables 1-10, we can note that when $n$ increases, the MLE and AvLe are decreased. 
Table 1: MLEs, estimates, $A 1, L 1, U 1$, and AvLe of the SIE model under TIIC for $x_{r}=0.8$ and $\theta=0.8$.

\begin{tabular}{|c|c|c|c|c|c|c|c|c|}
\hline \multirow{2}{*}{$n$} & \multirow{2}{*}{ MLE } & \multirow{2}{*}{$A 1$} & \multicolumn{3}{|c|}{$90 \%$} & \multicolumn{3}{|c|}{$95 \%$} \\
\hline & & & $L 1$ & U1 & AvLe & $L 1$ & U1 & AvLe \\
\hline 30 & 0.6394 & 0.0336 & 0.4777 & 0.8010 & 0.3233 & 0.4467 & 0.8320 & 0.3853 \\
\hline 50 & 0.6410 & 0.0327 & 0.5152 & 0.7667 & 0.2515 & 0.4911 & 0.7908 & 0.2997 \\
\hline 100 & 0.6454 & 0.0295 & 0.5474 & 0.7235 & 0.1761 & 0.5305 & 0.7403 & 0.2098 \\
\hline 200 & 0.6519 & 0.0294 & 0.5700 & 0.6939 & 0.1238 & 0.5582 & 0.7057 & 0.1476 \\
\hline 300 & 0.6530 & 0.0286 & 0.5824 & 0.6836 & 0.1013 & 0.5727 & 0.6933 & 0.1207 \\
\hline 500 & 0.6630 & 0.0284 & 0.5938 & 0.6722 & 0.0785 & 0.5863 & 0.6797 & 0.0935 \\
\hline 1000 & 0.6720 & 0.0282 & 0.6043 & 0.6597 & 0.0554 & 0.5990 & 0.6650 & 0.0660 \\
\hline 2000 & 0.6813 & 0.0276 & 0.6118 & 0.6509 & 0.0391 & 0.6080 & 0.6546 & 0.0466 \\
\hline
\end{tabular}

TAble 2: MLEs, estimates, $A 1, L 1, U 1$, and AvLe of the SIE model under TIIC for $x_{r}=0.9$ and $\theta=0.8$.

\begin{tabular}{|c|c|c|c|c|c|c|c|c|}
\hline \multirow{2}{*}{$n$} & \multirow{2}{*}{ MLE } & \multirow{2}{*}{$A 1$} & \multicolumn{3}{|c|}{$90 \%$} & \multicolumn{3}{|c|}{$95 \%$} \\
\hline & & & $L 1$ & $U 1$ & AvLe & $L 1$ & $U 1$ & AvLe \\
\hline 30 & 0.7151 & 0.0161 & 0.5501 & 0.9000 & 0.3500 & 0.5165 & 0.9336 & 0.4170 \\
\hline 50 & 0.7203 & 0.0125 & 0.5856 & 0.8550 & 0.2694 & 0.5598 & 0.8808 & 0.3210 \\
\hline 100 & 0.7270 & 0.0099 & 0.6222 & 0.8119 & 0.1897 & 0.6040 & 0.8301 & 0.2260 \\
\hline 200 & 0.7344 & 0.0090 & 0.6476 & 0.7813 & 0.1337 & 0.6348 & 0.7941 & 0.1592 \\
\hline 300 & 0.7359 & 0.0085 & 0.6594 & 0.7685 & 0.1090 & 0.6490 & 0.7789 & 0.1299 \\
\hline 500 & 0.7446 & 0.0079 & 0.6723 & 0.7569 & 0.0845 & 0.6642 & 0.7650 & 0.1007 \\
\hline 1000 & 0.7448 & 0.0076 & 0.6849 & 0.7447 & 0.0598 & 0.6791 & 0.7504 & 0.0713 \\
\hline 2000 & 0.7536 & 0.0056 & 0.6925 & 0.7347 & 0.0422 & 0.6885 & 0.7388 & 0.0503 \\
\hline
\end{tabular}

TABle 3: MLEs, estimates, $A 1, L 1, U 1$, and AvLe of the SIE model under TIIC for $x_{r}=1$ and $\theta=0.8$.

\begin{tabular}{|c|c|c|c|c|c|c|c|c|}
\hline \multirow{2}{*}{$n$} & \multirow{2}{*}{ MLE } & \multirow{2}{*}{$A 1$} & \multicolumn{3}{|c|}{$90 \%$} & \multicolumn{3}{|c|}{$95 \%$} \\
\hline & & & $L 1$ & $U 1$ & AvLe & $L 1$ & $U 1$ & AvLe \\
\hline 30 & 0.8200 & 0.0129 & 0.6229 & 0.9972 & 0.3743 & 0.5871 & 1.0330 & 0.4459 \\
\hline 50 & 0.8157 & 0.0073 & 0.6616 & 0.9498 & 0.2882 & 0.6341 & 0.9774 & 0.3434 \\
\hline 100 & 0.7893 & 0.0040 & 0.6983 & 0.9003 & 0.2021 & 0.6789 & 0.9197 & 0.2408 \\
\hline 200 & 0.7965 & 0.0017 & 0.7271 & 0.8699 & 0.1428 & 0.7134 & 0.8835 & 0.1701 \\
\hline 300 & 0.8024 & 0.0013 & 0.7438 & 0.8610 & 0.1171 & 0.7326 & 0.8722 & 0.1396 \\
\hline 500 & 0.7986 & 0.0008 & 0.7544 & 0.8448 & 0.0904 & 0.7457 & 0.8535 & 0.1077 \\
\hline 1000 & 0.8011 & 0.0004 & 0.7690 & 0.8331 & 0.0641 & 0.7629 & 0.8392 & 0.0763 \\
\hline 2000 & 0.8005 & 0.0002 & 0.7778 & 0.8231 & 0.0453 & 0.7735 & 0.8274 & 0.0539 \\
\hline
\end{tabular}

TABle 4: MLEs, estimates, $A 1, L 1, U 1$, and AvLe of the SIE model under TIIC for $x_{r}=0.8$ and $\theta=1.5$.

\begin{tabular}{|c|c|c|c|c|c|c|c|c|}
\hline \multirow{2}{*}{$n$} & \multirow{2}{*}{ MLE } & \multirow{2}{*}{$A 1$} & \multicolumn{3}{|c|}{$90 \%$} & \multicolumn{3}{|c|}{$95 \%$} \\
\hline & & & $L 1$ & $U 1$ & AvLe & $L 1$ & $U 1$ & AvLe \\
\hline 30 & 0.6394 & 0.0336 & 0.4777 & 0.8010 & 0.3233 & 0.4467 & 0.8320 & 0.3853 \\
\hline 50 & 0.6410 & 0.0327 & 0.5152 & 0.7667 & 0.2515 & 0.4911 & 0.7908 & 0.2997 \\
\hline 100 & 0.6454 & 0.0295 & 0.5474 & 0.7235 & 0.1761 & 0.5305 & 0.7403 & 0.2098 \\
\hline 200 & 0.6519 & 0.0294 & 0.5700 & 0.6939 & 0.1238 & 0.5582 & 0.7057 & 0.1476 \\
\hline 300 & 0.6530 & 0.0286 & 0.5824 & 0.6836 & 0.1013 & 0.5727 & 0.6933 & 0.1207 \\
\hline 500 & 0.6630 & 0.0284 & 0.5938 & 0.6722 & 0.0785 & 0.5863 & 0.6797 & 0.0935 \\
\hline 1000 & 0.6720 & 0.0282 & 0.6043 & 0.6597 & 0.0554 & 0.5990 & 0.6650 & 0.0660 \\
\hline 2000 & 0.6813 & 0.0276 & 0.6118 & 0.6509 & 0.0391 & 0.6080 & 0.6546 & 0.0466 \\
\hline
\end{tabular}

\section{Results and Discussion}

4.1. Application. In this section, two real datasets are analyzed to explain the benefit of the SIE model compared to the IE model. To compare the competitive models, we suggested some information criterion (ICr) as minus two log-LLFu
(D1), Akaike ICr (D2), the correct Akaike ICr (D3), Bayesian ICr (D4), and Hannan-Quinn ICr (D5).

The first data are known as ball bearing data, and it represents the number of rotations before ball bearing failure obtained [18]. The second data set consists of 100 observations of breaking stress of carbon fibres (in Gba) given by 
Table 5: MLEs, estimates, $A 1, L 1, U 1$, and AvLe of the SIE model under TIIC for $x_{r}=0.9$ and $\theta=1.5$.

\begin{tabular}{|c|c|c|c|c|c|c|c|c|}
\hline \multirow{2}{*}{$n$} & \multirow{2}{*}{ MLE } & \multirow{2}{*}{$A 1$} & \multicolumn{3}{|c|}{$90 \%$} & \multicolumn{3}{|c|}{$95 \%$} \\
\hline & & & $L 1$ & U1 & AvLe & $L 1$ & $U 1$ & AvLe \\
\hline 30 & 0.7151 & 0.0161 & 0.5501 & 0.9000 & 0.3500 & 0.5165 & 0.9336 & 0.4170 \\
\hline 50 & 0.7203 & 0.0125 & 0.5856 & 0.8550 & 0.2694 & 0.5598 & 0.8808 & 0.3210 \\
\hline 100 & 0.7270 & 0.0099 & 0.6222 & 0.8119 & 0.1897 & 0.6040 & 0.8301 & 0.2260 \\
\hline 200 & 0.7344 & 0.0090 & 0.6476 & 0.7813 & 0.1337 & 0.6348 & 0.7941 & 0.1592 \\
\hline 300 & 0.7359 & 0.0085 & 0.6594 & 0.7685 & 0.1090 & 0.6490 & 0.7789 & 0.1299 \\
\hline 500 & 0.7446 & 0.0079 & 0.6723 & 0.7569 & 0.0845 & 0.6642 & 0.7650 & 0.1007 \\
\hline 1000 & 0.7448 & 0.0076 & 0.6849 & 0.7447 & 0.0598 & 0.6791 & 0.7504 & 0.0713 \\
\hline 2000 & 0.7536 & 0.0056 & 0.6925 & 0.7347 & 0.0422 & 0.6885 & 0.7388 & 0.0503 \\
\hline
\end{tabular}

TABle 6: MLEs, estimates, $A 1, L 1, U 1$, and AvLe of the SIE model under TIIC for $x_{r}=1$ and $\theta=1.5$.

\begin{tabular}{|c|c|c|c|c|c|c|c|c|}
\hline \multirow{2}{*}{$n$} & \multirow{2}{*}{ MLE } & \multirow{2}{*}{$A 1$} & \multicolumn{3}{|c|}{$90 \%$} & \multicolumn{3}{|c|}{$95 \%$} \\
\hline & & & $L 1$ & $U 1$ & AvLe & $L 1$ & $U 1$ & AvLe \\
\hline 30 & 0.8200 & 0.0129 & 0.6229 & 0.9972 & 0.3743 & 0.5871 & 1.0330 & 0.4459 \\
\hline 50 & 0.8157 & 0.0073 & 0.6616 & 0.9498 & 0.2882 & 0.6341 & 0.9774 & 0.3434 \\
\hline 100 & 0.7893 & 0.0040 & 0.6983 & 0.9003 & 0.2021 & 0.6789 & 0.9197 & 0.2408 \\
\hline 200 & 0.7965 & 0.0017 & 0.7271 & 0.8699 & 0.1428 & 0.7134 & 0.8835 & 0.1701 \\
\hline 300 & 0.8024 & 0.0013 & 0.7438 & 0.8610 & 0.1171 & 0.7326 & 0.8722 & 0.1396 \\
\hline 500 & 0.7986 & 0.0008 & 0.7544 & 0.8448 & 0.0904 & 0.7457 & 0.8535 & 0.1077 \\
\hline 1000 & 0.8011 & 0.0004 & 0.7690 & 0.8331 & 0.0641 & 0.7629 & 0.8392 & 0.0763 \\
\hline 2000 & 0.8005 & 0.0002 & 0.7778 & 0.8231 & 0.0453 & 0.7735 & 0.8274 & 0.0539 \\
\hline
\end{tabular}

Table 7: MLEs, estimates, $A 1, L 1, U 1$, and AvLe of the SIE model under TIC for $T=1.5$ and $\theta=0.2$.

\begin{tabular}{|c|c|c|c|c|c|c|c|c|}
\hline \multirow{2}{*}{$n$} & \multirow{2}{*}{ MLE } & \multirow{2}{*}{$A 1$} & \multicolumn{3}{|c|}{$90 \%$} & \multicolumn{3}{|c|}{$95 \%$} \\
\hline & & & $L 1$ & $U 1$ & AvLe & $L 1$ & U1 & AvLe \\
\hline 30 & 0.1671 & 0.0040 & 0.1252 & 0.2090 & 0.0838 & 0.1172 & 0.2170 & 0.0998 \\
\hline 50 & 0.1672 & 0.0033 & 0.1350 & 0.1995 & 0.0645 & 0.1288 & 0.2056 & 0.0769 \\
\hline 100 & 0.1674 & 0.0027 & 0.1444 & 0.1898 & 0.0455 & 0.1400 & 0.1942 & 0.0542 \\
\hline 200 & 0.1685 & 0.0018 & 0.1533 & 0.1857 & 0.0324 & 0.1502 & 0.1888 & 0.0386 \\
\hline 300 & 0.1688 & 0.0017 & 0.1557 & 0.1820 & 0.0263 & 0.1531 & 0.1845 & 0.0314 \\
\hline 500 & 0.1696 & 0.0012 & 0.1575 & 0.1778 & 0.0203 & 0.1555 & 0.1797 & 0.0242 \\
\hline 1000 & 0.1714 & 0.0010 & 0.1641 & 0.1787 & 0.0146 & 0.1627 & 0.1801 & 0.0174 \\
\hline 2000 & 0.1719 & 0.0008 & 0.1667 & 0.1771 & 0.0103 & 0.1658 & 0.1781 & 0.0123 \\
\hline
\end{tabular}

Table 8: MLEs, estimates, $A 1, L 1, U 1$, and AvLe of the SIE model under TIC for $T=3$ and $\theta=0.2$.

\begin{tabular}{|c|c|c|c|c|c|c|c|c|}
\hline \multirow{2}{*}{$n$} & \multirow{2}{*}{ MLE } & \multirow{2}{*}{$A 1$} & \multicolumn{3}{|c|}{$90 \%$} & \multicolumn{3}{|c|}{$95 \%$} \\
\hline & & & $L 1$ & U1 & AvLe & $L 1$ & U1 & AvLe \\
\hline 30 & 0.1898 & 0.0051 & 0.1447 & 0.2349 & 0.0902 & 0.1360 & 0.2435 & 0.1075 \\
\hline 50 & 0.1913 & 0.0032 & 0.1591 & 0.2295 & 0.0704 & 0.1523 & 0.2362 & 0.0839 \\
\hline 100 & 0.1923 & 0.0024 & 0.1668 & 0.2159 & 0.0491 & 0.1621 & 0.2206 & 0.0585 \\
\hline 200 & 0.1925 & 0.0021 & 0.1760 & 0.2110 & 0.0350 & 0.1727 & 0.2143 & 0.0417 \\
\hline 300 & 0.1931 & 0.0019 & 0.1788 & 0.2073 & 0.0285 & 0.1761 & 0.2100 & 0.0340 \\
\hline 500 & 0.1939 & 0.0016 & 0.1828 & 0.2050 & 0.0222 & 0.1807 & 0.2071 & 0.0264 \\
\hline 1000 & 0.1940 & 0.0016 & 0.1828 & 0.2050 & 0.0222 & 0.1807 & 0.2071 & 0.0264 \\
\hline 2000 & 0.1942 & 0.0012 & 0.1864 & 0.2020 & 0.0157 & 0.1849 & 0.2035 & 0.0186 \\
\hline
\end{tabular}


TABle 9: MLEs, estimates, $A 1, L 1, U 1$, and AvLe of the SIE model under TIC for $T=1.5$ and $\theta=0.8$.

\begin{tabular}{|c|c|c|c|c|c|c|c|c|}
\hline \multirow{2}{*}{$n$} & \multirow{2}{*}{ MLE } & \multirow{2}{*}{$A 1$} & \multicolumn{3}{|c|}{$90 \%$} & \multicolumn{3}{|c|}{$95 \%$} \\
\hline & & & $L 1$ & $U 1$ & AvLe & $L 1$ & $U 1$ & AvLe \\
\hline 30 & 0.6316 & 0.0335 & 0.4710 & 0.7921 & 0.3211 & 0.4403 & 0.8229 & 0.3826 \\
\hline 50 & 0.6323 & 0.0314 & 0.5081 & 0.7565 & 0.2484 & 0.4843 & 0.7803 & 0.2959 \\
\hline 100 & 0.6382 & 0.0311 & 0.5408 & 0.7156 & 0.1748 & 0.5241 & 0.7324 & 0.2083 \\
\hline 200 & 0.6403 & 0.0306 & 0.5684 & 0.6922 & 0.1238 & 0.5565 & 0.7040 & 0.1475 \\
\hline 300 & 0.6481 & 0.0302 & 0.5777 & 0.6784 & 0.1007 & 0.5680 & 0.6881 & 0.1200 \\
\hline 500 & 0.6494 & 0.0294 & 0.5903 & 0.6685 & 0.0782 & 0.5828 & 0.6760 & 0.0931 \\
\hline 1000 & 0.6578 & 0.0288 & 0.6003 & 0.6554 & 0.0551 & 0.5950 & 0.6607 & 0.0657 \\
\hline 2000 & 0.6583 & 0.0276 & 0.6088 & 0.6478 & 0.0390 & 0.6050 & 0.6515 & 0.0465 \\
\hline
\end{tabular}

TAble 10: MLEs, estimates, $A 1, L 1, U 1$, and AvLe of the SIE model under TIC for $T=3$ and $\theta=0.8$.

\begin{tabular}{|c|c|c|c|c|c|c|c|c|}
\hline \multirow{2}{*}{$n$} & \multirow{2}{*}{ MLE } & \multirow{2}{*}{$A 1$} & \multicolumn{3}{|c|}{$90 \%$} & \multicolumn{3}{|c|}{$95 \%$} \\
\hline & & & $L 1$ & $U 1$ & AvLe & $L 1$ & $U 1$ & AvLe \\
\hline 30 & 0.7492 & 0.0117 & 0.5783 & 0.9400 & 0.3617 & 0.5437 & 0.9747 & 0.4310 \\
\hline 50 & 0.7575 & 0.0080 & 0.6099 & 0.8852 & 0.2753 & 0.5835 & 0.9115 & 0.3280 \\
\hline 100 & 0.7639 & 0.0063 & 0.6470 & 0.8409 & 0.1939 & 0.6284 & 0.8594 & 0.2310 \\
\hline 200 & 0.7646 & 0.0045 & 0.6760 & 0.8133 & 0.1373 & 0.6629 & 0.8264 & 0.1635 \\
\hline 300 & 0.7738 & 0.0041 & 0.6878 & 0.7998 & 0.1120 & 0.6771 & 0.8105 & 0.1334 \\
\hline 500 & 0.7748 & 0.0040 & 0.6878 & 0.7998 & 0.1120 & 0.6771 & 0.8105 & 0.1334 \\
\hline 1000 & 0.7807 & 0.0039 & 0.6994 & 0.7860 & 0.0866 & 0.6911 & 0.7943 & 0.1031 \\
\hline 2000 & 0.7820 & 0.0037 & 0.7115 & 0.7726 & 0.0611 & 0.7056 & 0.7785 & 0.0729 \\
\hline
\end{tabular}

TABle 11: MLEs, D1, D2, D3, D4, and D5 for ball bearing data.

\begin{tabular}{lcccccc}
\hline Models & MLE and SE & $D 1$ & $D 2$ & $D 3$ & $D 4$ & D5 \\
\hline SIE & $73.586(11.48)$ & 243.07 & 245.07 & 245.252 & 244.45 & 245.382 \\
IE & $20.403(2.55)$ & 253.927 & 255.927 & 256.109 & 255.307 & 256.24 \\
\hline
\end{tabular}

TABLE 12: MLEs, D1, D2, D3, D4, and D5 for carbon fibres' data.

\begin{tabular}{lcccccc}
\hline Models & MLE and SE & $D 1$ & $D 2$ & $D 3$ & $D 4$ & D5 \\
\hline SIE & $2.817(11.48)$ & 348.384 & 350.384 & 350.404 & 350.384 \\
IE & $2.135(2.55)$ & 397.956 & 399.956 & 399.977 & 399.956 & 401.011 \\
\hline
\end{tabular}

[19]. According to Tables 11 and 12, our new model is better suited than the IE model and has the lowest values for all statistics.

4.2. Discussion. From the modelling to ball bearing and carbon fibres datasets, we see that the SIE model provides the greatest fit for the both datasets. The numerical values in Tables 11 and 12 are proposed; the both datasets supported the superiority of the SIE model.

\section{Conclusion}

In this study, we proposed a new one-parameter model, which is called the SIE model. Some basic statistical properties of the SIE model are also proposed. Estimation of the SIE parameter was assessed by using the ML method of estimation censored and complete samples. Application to carbon fibres datasets were used to explain the importance of SIE model against the IE model. The SIE model as we see is very flexible and simple model, so many authors can use it in the future articles. The authors can use Bayesian estimation under complete and various censored schemes to estimate its parameters. Also, ranked set sampling papers can apply the new model. Also, the authors which are interested in distribution theory can generalize more extensions of this model by many different ways.

\section{Data Availability}

The data used to support the findings of the study are available from the corresponding author upon request.

\section{Conflicts of Interest}

The authors declare no conflicts of interest.

\section{Acknowledgments}

This study was supported by King Saud University, Deanship of Scientific Research, College of Science Research Center. 


\section{References}

[1] R. Amirov, A. Ergun, and S. Durak, "Half-inverse problems for the quadratic pencil of the Sturm-Liouville equations with impulse," Numerical Methods for Partial Differential Equations, vol. 37, no. 1, pp. 915-924, 2021.

[2] R. K. Amirov and A. Ergun, "Half inverse problems for the impulsive singular diffusion operator," Turkish Journal of Science, vol. 5, no. 3, pp. 186-198, 2020.

[3] A. Ergün, "A half-inverse problem for the singular diffusion operator with jump conditions," Miskolc Mathematical Notes, vol. 21, no. 2, pp. 805-821, 2020.

[4] G. M. Cordeiro and M. de Castro, "A new family of generalized distributions," Journal of Statistical Computation and Simulation, vol. 81, no. 7, pp. 883-898, 2011.

[5] D. Kumar, U. Singh, and S. K. Singh, "A new distribution using sine function- its application to bladder cancer patients data," Journal of Statistics Applications \& Probability, vol. 4, no. 3, pp. 417-427, 2015.

[6] S. A. Elsherpieny and M. Elsehetry, "Kumaraswamy type I half logistic family of distributions with applications," Gazi University Journal of Science, vol. 32, no. 1, pp. 333-349, 2019.

[7] M. Bourguignon, R. B. Silva, and G. M. Cordeiro, "The Weibull-G family of probability distributions," Journal of Data Science, vol. 12, pp. 53-68, 2014.

[8] M. A. Haq and M. Elgarhy, "The odd Fréchet-G family of probability distributions," Journal of Statistics Applications and Probability, vol. 7, pp. 185-201, 2018.

[9] H. M. Yousof, A. Z. Afify, G. G. Hamedani, and G. Aryal, "The burr X generator of distributions for lifetime data," Journal of Statistical Theory and Applications, vol. 16, pp. 1-19, 2017.

[10] A. A. Mohamed, R. M. Mahmoud, and S. A. ElSherbini, "The new Kumaraswamy Kumaraswamy family of generalized distributions with application," Pakistan Journal of Statistics and Operation Research, vol. 11, no. 2, pp. 159-180, 2015.

[11] M. A. Aldahlan, F. Jamal, C. Chesneau, M. Elgarhy, and I. Elbatal, "The truncated Cauchy power family of distributions with inference and applications," Entropy, vol. 22, pp. 1-25, 2020.

[12] G. M. Cordeiro, M. Alizadeh, T. G. Ramires, and E. M. M. Ortega, "The generalized odd half-Cauchy family of distributions: properties and applications," Communications in Statistics-Theory and Methods, vol. 46, no. 11, pp. 56855705, 2017.

[13] P. E. Oguntunde, A. O. Adejumo, and E. A. Owoloko, "Exponential inverse exponential (EIE) distribution with applications to lifetime data," Asian Journal of Scientific Research, vol. 10, no. 3, pp. 169-177, 2017.

[14] P. E. Oguntunde, M. A. Khaleel, A. O. Adejumo, H. I. Okagbue, A. A. Opanuga, and F. O. Owolabi, "The Gompertz inverse exponential (GoIE) distribution with applications," Cogent Mathematics \& Statistics, vol. 5, no. 1, Article ID 1507122, 2018.

[15] P. E. Oguntunde, A. O. Adejumo, and E. A. Owoloko, "Application of Kumaraswamy inverse exponential distribution to real lifetime data," International Journal of Applied Mathematics \& Statistics, vol. 56, no. 5, pp. 34-47, 2017.

[16] M. A. Aldahlan, "The inverse Weibull inverse exponential distribution with application," International Journal of Contemporary Mathematical Sciences, vol. 14, no. 1, pp. 17-30, 2019.

[17] H. L. MacGillivray, "Skewness and asymmetry: measures and orderings," Annals of Statistics, vol. 14, pp. 994-1011, 1986.
[18] M. J. Crowder, A. C. Kimber, R. L. Smith, and T. J. Sweeting, Statistical Analysis of Reliability Data, Chapman \& Hall, London, UK, 1991.

[19] M. D. Nichols and W. J. Padgett, "A bootstrap control chart for Weibull percentiles," Quality and Reliability Engineering International, vol. 22, no. 2, pp. 141-151, 2006. 